\title{
PLANEAMIENTO ESTRATÉGICO APLICADO A LAS MICRO Y PEQUEÑAS EMPRESAS EN LA REGIÓN LIMA-PROVINCIAS, 2016
}

\author{
STRATEGIC PLANNING APPLIED TO MICRO AND SMALL ENTERPRISES \\ IN THE LIMA PROVINCES REGION, 2016
}

José García Paredes ${ }^{1}$, Jesús Jacobo Coronado Espinoza²

\section{RESUMEN}

Objetivo: Conocer el nivel de aplicación del planeamiento estratégico en las MYPES de la Región LimaProvincias en el 2016. Material y Método: Estudio descriptivo, no experimental y transversal, se encuestó a una muestra de 367 MYPES utilizando el cuestionario y la entrevista, el instrumento para la toma de datos fue validado y confiable procedente del juicio de expertos y pruebas piloto. Resultados: E1 20\% de las MYPES aplican el planeamiento estratégico, principalmente las ubicadas en la franja costera. Conclusión: La situación encontrada revela una escasa utilización formal del planeamiento estratégico por la parte de las MYPES en el ámbito de estudio asociada a la poca cultura empresarial coexistente con un bajo nivel de competitividad en los negocios.

Palabras clave: Planeamiento estratégico; Financiamiento, Producción y mercado.

\section{ABSTRACT}

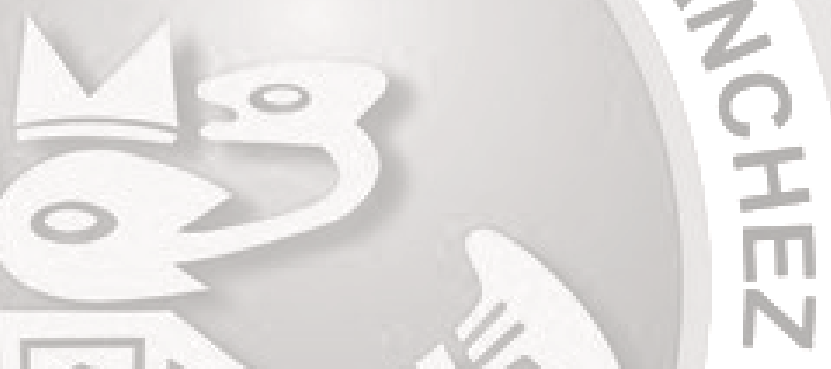

Objective: To know the level of application of strategic planning in the MYPES of the Lima-Provincias Region in the 2016 period. Materials and Methods: The descriptive, non-experimental and transversal study method was surveyed to a sample of 367 MYPES using the questionnaire and the interview, The instrument for data collection was validated and reliable from expert judgment and pilot tests. Results: $20 \%$ of the MYPES apply strategic planning, mainly those located in the coastal strip. Conclusions: The situation found reveals a low formal use of strategic planning by the part of the MYPES in the field of study associated with the little business culture coexisting with a low level of competitiveness in business.

Key words: Strategic planning, Financing, Production and Market.

'Docente de la Facultad de Ciencias Empresariales - UNJFSC - Maestro en Marketing Agroalimentario. Email: jusho56@gmail.com

${ }^{2}$ Docente de la Facultad de Ciencias Empresariales - UNJFSC - Doctor Administrativo. Email : jjcoril@hotmail.com 


\section{INTRODUCCIÓN}

A la fecha, y por muy largo tiempo, imparablemente el proceso de globalización económica que hace que los mercados sean cada vez más competitivos con requerimientos constantes en la mejora contínua de la calidad de los bienes y servicios, que junto a tendencias de precios cada vez más accesibles o cómodos, exigen pues productos competitivos y posicionamiento de mercado. Contexto en el cual actualmente obliga a la empresa a adoptar las estrategias pertinentes para acceder y mantenerse en el mercado, constituyéndose en esta forma un serio reto para las MYPES.

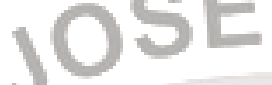

Por otro lado, como reflejo de las características de las MYPES en el país, en la Región Lima, también su presencia es relevante en razón a que conforman una parte importante de la base económica regional así como la generación de empleo; demostrando con ello la creatividad y capacidades de sus propietarios, sin embargo, sus áctividades de tipo empresarial responden fundamentalmente al empirismo, con una clara presencia de una muy escasa utilización y/o aplicación del planeamiento estratégico formal. Aspecto que convive con un limitado e insuficiente apoyo por parte de las entidades públicas, privadas y del mundo académico.

También, es necesario considerar que las MYPES son más del $50 \%$ de las unidades productivas de la Región Lima, desarrollan actividades que en orden de importancia son las extractivas (agricultura, ganadería, caza, pesca y minería) y donde se encuentra la mayor PEA, seguido de la transformación (industria manufacturera, artesanía y construcción, comercio y servicios). Éstas carecen de capacitación, limitado acceso al financiamiento, dificultades en su comercialización y no son competitivas; característica que es compartida con la pequeña empresa individual. También los operadores turísticos se encuentran poco desarrollados en función a las limitaciones de la inversión pública y escasa cultura turística. No obstante, las MYPES son un sector con enormes posibilidades de competitividad y dinamismo por su capacidad de adaptación para atender a segmentos específicos y diferenciados de mercado, así como por su flexibilidad frente a los cambios y su capacidad de innovación. Sin embargo, se destaca, la actividad industrial con PYMES con presencia en las provincias de Huaura, Huaral,
Barranca y Cañete en razón a factores de localización de infraestructura: puertos, carreteras, servicios, etc. En este sector las MYPES no se incrementaron, siendo muy heterogéna y dinámica con deficiencias en conocimiento de mercado, precios y Tecnología, gestión empresarial y comercialización de sus productos, financiamiento y alta informalidad.

La densidad empresarial en la Región Lima es de 50,8 por cada mil habitantes, muy por debajo de 61 empresas a nivel nacional, siendo las provincias de Barranca, Cañete, Huaura y Huaral las de mayor una participãción; de lo cual se estima que en promedio un $80 \%$ corresponden a las MYPES. Lo que indica que aún hay mucho por crecer y expandir sus actividades dada la disponibilidad de recursos de la zona.

La situación descrita de las MYPES en las provincias de la Región Lima, dada su importancia económica y social, están llamada a dar una respuesta ante un mundo en permanente cambio que al mismo tiempo ofrece oportunidades comerciales, económicas, financieras, tecnológicas, apertura de mercados, etc. Pero para ello las MYPES deben estar a la altura de dichas exigencias, que como punto de partida debe aplicarse el planeamiento estratégico.

El planeamiento estratégico es un instrumento de gestión que si se aplicaría en las MYPES de las provincias de la Región Lima impactaría competitivamente en el stock y flujo empresarial, así como elevaría la densidad empresarial; motivo por el cual amerita formularse la siguiente interrogante: ¿En qué proporción se aplica el planeamiento estratégico en las MYPES de la Región Lima en el 2016?

Frente a la problemática del contexto antes mencionado, la presente investigación sobre el "Planeamiento estratégico aplicado a las micro y pequeñas empresas en la Región Lima - Provincias, 2016" pretende contribuir a resolver un problema económico y social. Aspecto que se justifica por su conveniencia en la medida que servirá posteriormente para orientar acciones y políticas de apoyo y promoción del crecimiento y desarrollo de las MYPES, por su relevancia social toda vez que beneficiará a un importante sector poblacional, y por su implicancia práctica en razón a su aporte a la solución de diferentes problemas de carácter empresarial de las MYPES. 
Al respecto, por ejemplo, Farías, (2016) en su tesis de maestría titulada "El uso de la planeación estratégica para desarrollar una PYME en México", señala los comunes problemas de una pequeña empresa relacionados con la carencia de un FODA, objetivos, desorganización, sin visión, ni visión, sin políticas ni valores, sin planes estratégicos; que permitan un mejor rendimiento. Esto explica su alta mortandad. La autora toma un caso de aplicación del planeamiento estratégico donde como objetivos específicos de la empresa se fijan: alcanzar una rentabilidad del $50 \%$ en los próximos dos años, aumentar la cartera de clientes cinco veces de la actual y aumentar las ventas en $30 \%$ en lo que resta del año. Que luego de aplicar el planeamiento estratégico y un elemental plan de negocios para una vida útil de 10 años, se obtuvieron indicadores económico - financieros positivos expresados en el valor actual neto (VAN), la tasa interna de retorno o rentabilidad (TIR) una recuperación de la inversión en el corto plazo; aún considerando un análisis de sensibilidad de un aumento del $10 \%$ en sus costos y una reducción de sus ingresos en la misma proporción.

También, Rosas (2016), en su tesis de maestría titulada "Planeamiento estratégico y sus repercusiones en la gestión empresarial del sector turismo de la Región de Puno 2015-2016", como objetivo explica dichas repercusiones bajo una investigación explicativa con diseño no experimental, en una muestra de 100 empresas turísticas; cuyos resultados fueron: el nivel de planeamiento estratégico existe un $44 \%$ en nivel regular, un $29 \%$ de nivel no óptimo y un $27 \%$ presenta un nivel óptimo; en la gestión empresarial se afirma que el $46 \%$ tuvieron un nivel regular, un $43 \%$ de nivel bueno, $11 \%$ con nivel deficiente; en la dimensión conocimiento, el 54\% tuvo un nivel regular, un $35 \%$ un nivel no óptimo, un $11 \%$ un nivel óptimo; en la dimensión de participación, el $49 \%$ un nivel óptimo, $37 \%$ un nivel regular, el $14 \%$ tuvo un nivel no óptimo; en la dimensión evaluación, se evidencia que el $73 \%$ tuvieron un nivel no óptimo, un $25 \%$ de empresas tuvieron un nivel regular, un $2 \%$ un nivel óptimo.

Concluyendo que existe una correlación directa y significativa $(r=0,220)$ entre el planeamiento estratégico y la gestión empresarial, en la dimensión conocimiento y la gestión empresarial $(\mathrm{r}=0,358)$, en la dimensión participación y la gestión empresarial $(\mathrm{r}=.466)$, el planeamiento estratégico en la dimensión evaluación y la gestión empresarial $(\mathrm{r}=$ .227).

Según Girio, (2012) en su tesis de pregrado titulada: "Diseño Organizacional en las Micro y Pequeñas Empresas registradas en la Cámara de comercio y Producción de la Provincia de Huaura" Concluye:

(a) La empresa en su mayoría representada por un $78 \%$, el modelo genérico de estructura organizacional según precepción de sus gerentes y/o dueños es mecanicista con un control regido por parte de la autoridad y poca atención a la ejecución de tareas de manera individual. (b) Son pocos frecuentes los diseños de estructura organizacionales orgánicas y adaptivas, y representan en un $2.4 \%$ el diseño de estructura orgánica, el $33.1 \%$ el diseño de estructura adaptativa. (c) La responsabilidad de gestión en las micro empresas y pequeñas empresas registradas en la cámara de comercio y producción de la provincia de Huaura, está en manos del Gerente - Dueño en $35 \%$, del Gerente en un $53 \%$ y del dueño en un $11 \%$.

Dichas conclusiones, evidencian una vez más, una escasa utilización del planeamiento estratégico como instrumento que permita o facilite el alcance de la competitividad empresarial.

Por lo visto, pues, es indudable e impostergable que el planeamiento estratégico, que en el mundo académico se le denomina también dirección estratégica, sea pues aplicado en las MYPES de la Región Lima y mejorar su nivel de competitividad.

En este sentido, según los autores, la dirección estratégica o planeamiento estratégico se ha convertido en un paradigma dominante para comprender las actividades empresariales en un medio sumamente difícil, para aprovechar las oportunidades y de alguna manera cubrirse de las amenazas en función a sus capacidades y potencialidades en razón a sus fortalezas y minimizando sus debilidades. De ello depende y nace la ruta coexistente con el establecimiento de diferentes alternativas conducentes hacia el alcance de objetivos.(Guerra y Navas, 2015), p.23-24.

Por su parte, en línea a lo señalado, el autor menciona que la planeación estratégica es el proceso mediante el cual quienes toman decisiones en una organización obtienen, procesan y analizan información pertinente interna y externa, con el fin de evaluar la situación presente de la empresa, así como de su nivel de competitividad con el propósito 
de anticipar y decidir sobre el direccionamiento de la institución hacia el futuro.

(Amaya, 2005, p. 14).

Es obvio que ello significa conocer, a través de un diagnóstico, es decir mediante un análisis FODA, la situación actual del negocio, para luego determinar objetivos alcanzables, estableciendo metas específicas que constituyen las rutas seguir y la forma de cómo hacerlo.

Al respecto, haciendo referencia a la administración estratégica, Robbins y Couther (2014) mencionan que "son los planes que determinan cómo lograr su propósito comercial, cómo competir con éxito y cómo atraerá y satisfará a sus clientes para cumplir sus objetivos" (p.240).

El autor, considera a la planificación estratégica como sinónimo de la dirección estratégica que comprende la visión, misión, el análisis FODA, el establecimiento de objetivos y estrategias, como un proceso integrado en el tiempo sujeta a una contínua revisión y evaluación de los factores internos y externos de la empresa; en cuyo contexto se integra la gerencia, la mercadotecnia, las finanzas, la contabilidad, la producción, las operaciones la investigación y desarrollo, así como los sistemas informáticos, para generar nuevas oportunidades en razón a las nuevas tendencias. Para ello, tomando permanentemente decisiones que conllevan a la posición futura de los negocios de acuerdo al entorno y las circunstancias por la que atraviesa, considerando la gestión del talento humano y la disponibilidad de sus factores productivos. (David, F. 2003,pp. xxv, xxvi, 5 y 6 ).

Por lo expuesto, el presente trabajo establece como objetivo general: identificar la proporción en que se aplica la planeación estratégica en las MYPES de la Región Lima - Provincias en el periodo 2016. Tomando como dimensiones al aspecto financiero, la producción y tecnología, y el mercado. Componentes de vital importancia porque de ello depende la competitividad de las MYPES.

\section{MATERIALESY MÉTODOS}

Método: El estudio corresponde a una investigación de tipo descriptivo, no experimental; tuvo por finalidad describir el fenómeno o la situación en una circunstancia establecida de forma temporal espacial determinada.

Ámbito territorial: la investigación se realizó en las provincias de la Región Lima, con énfasis en la zona costera por concentrar la mayor actividad económica

\section{Población: 1750 MYPES formales}

Muestra: 367 MYPES, determinada por conveniencia, distribuida en orden de importancia del siguiente modo:

Tabla 1. Distribución de la muestra según provincias de la Región Lima

\begin{tabular}{lc}
\hline PROVINCIAS & $\%$ \\
\hline Huarochiri & 5 \\
Oyon & 4 \\
Cajatambo & 2 \\
Yauyos & 2 \\
Huaura & 25 \\
Cañete & 22 \\
Huaral & 19 \\
Barranca & 19 \\
Canta & 100,0 \\
Total & \\
\hline
\end{tabular}

Técnicas de recopilación de datos: la encuesta y la entrevista

Técnicas de procesamiento de información: estadística descriptiva, escala de Likert, Software estadístico SPSS para Windows v.20

Materiales utilizados: escritorio, Computadora, Impresora, útiles de escritorio diversos,

\section{RESULTADOS}

De acuerdo a la información analizada y depurada, se obtuyo los siguientes resultados:

a. En general en las nueve provincias indicadas para el período investigado, bajo los términos de la aplicación de un planeamiento estratégico formal en las dimensiones consideradas, no es aplicado en un 60\% de las MYPES, reflejando una situación de aplicación neutral un $20 \%$, y siendo aplicado solamente en un $20 \%$ de las mismas, las que son mayormente pequeñas empresas ubicadas en la franja costera 
b. En relación al planeamiento estratégico para las finanzas, del total de las MYPES, el 61\% arroja una situación de no aplicación, un 19\% una situación neutral y el $20 \%$ restante presenta una situación de aplicación aceptable.

c. Por su parte el planeamiento estratégico para la producción y tecnología, del total de las MYPES, no es aplicado en un $60 \%$, teniendo una situación de aplicación neutral en un $22 \%$ y solo es aplicado por el $18 \%$.

d. En cuanto a la adopción del planeamiento estratégico formal utilizado en el mercado y marketing, del total de las MYPES, el 59\% refleja una situación de no aplicación, un 20\% una situación de aplicación neutral y solo es aplicado en el $21 \%$ restante.

Tabla 2. Estado Situacional sobre la aplicación del planeamiento estratégico y competitividad empresarial de las MYPES en la Región Lima

\begin{tabular}{lccc}
\hline & \multicolumn{3}{c}{} \\
\cline { 2 - 4 } & SYtuación de & Situación de & Situación de \\
Planeamiento Formal & Aplicación & Aplicación & Aplicación no \\
& Aceptable & Neutral & Aceptable \\
\hline Financiero & $20 \%$ & $19 \%$ & $61 \%$ \\
Producción y Tecnología & $18 \%$ & $22 \%$ & $60 \%$ \\
Mercado y Marketing & $21 \%$ & $20 \%$ & $59 \%$ \\
Total Región & $20 \%$ & $20 \%$ & $60 \%$ \\
\hline
\end{tabular}

\section{DISCUSIÓN}

Los resultados son congruente con los encontrados en los antecedentes, por ejemplo, con Farías (2016) además de coincidir con las dificultades que poseen las MYPES, requieren del planeamiento estratégico para alcanzar rentabilidad y mejora en sus ventas; también, los resultados de la presenta investigación se asemeja con los hallazgos de Rosas, R. (2016) y de Girio, E. (2012), en la medida que el planeamiento estratégico finalmente se evalúa en resultados expresados en financiamiento, producción y tecnología y posicionamiento de mercado.

Muchos estudios se han realizado en el país sobre las MYPES, que en el caso de las provincias de la Región Lima se observó una escasa presencia de la cultura empresarial manifestada a través de la reducida utilización del planeamiento estratégico formal como un documento de gestión y orientador sobre el rumbo en el mediano y largo plazo según el sector económico al que pertenecen dichas MYPES, aspecto que hoy en día se exige en todo mercado competitivo.

Empero, los micros y pequeños empresarios de la Región Lima no dejan de lado la consideración acerca de la importancia de la aplicación del
Planeamiento Estratégico en el manejo empresarial ya que les permitiría orientar todas sus decisiones a la ruta estratégica de mediano y largo plazo.

\section{CONCLUSIÓN}

Existe un fuerte predominio de una no relevante utilización o aplicación del planeamiento estratégico formal en las MYPES en la zona de estudio, que, al juzgar por las dimensiones indicadas como componentes por excelencia de un planeamiento estratégico, explican sus bajos niveles de competitividad empresarial; aspecto que estaría limitando su acceso a las nuevas tendencias y oportunidades comerciales, y por ende restringen su crecimiento y desarrollo. Excepto aquellas pequeñas empresas costeras vinculadas a la agroexportación, agroindustria, minería, pesca, hotelería y restaurantes categorizados, entre otros.

\section{AGRADECIMIENTOS}

Se agradece al equipo de investigación y a los alumnos de la facultad de Ciencia s Empresariales así como a las micro y pequeñas empresas que apoyaron en la aplicación de las encuestas. 


\section{REFERENCIAS BIBLIOGRÁFICAS}

Amaya, J. A. (2005). Planeación y Estrategia. Bucaramanga. Universidad Santo Tomás. A. Francés (2006) Planeamiento y Planes para la Empresa (1ra. Ed.) Mexico: Pearson

D'Alessio, F. (2014). Planeamiento Estratégico Razonado. Aspectos Conceptuales y

David, F. (2003): Conceptos de Administración Estratégica. (9na Ed.) Mexico: Pearson

Farías, E. (2016). El uso de la planeación estratégica para desarrollar una PYME en México (tesis de mestría). Instituto Politécnico Nacional-Escuela Superior de Ingeniería Mecánica y Eléctrica. México 2016.

Guerras M.L.A \& Navas L.J.C.(2015). La direccion estrategica de la empresa. teoria y aplicaciones. Thommson Reuteres. civita $5^{\text {TA }}$ edicion Editorial Aranzadi S.A.Madrid..

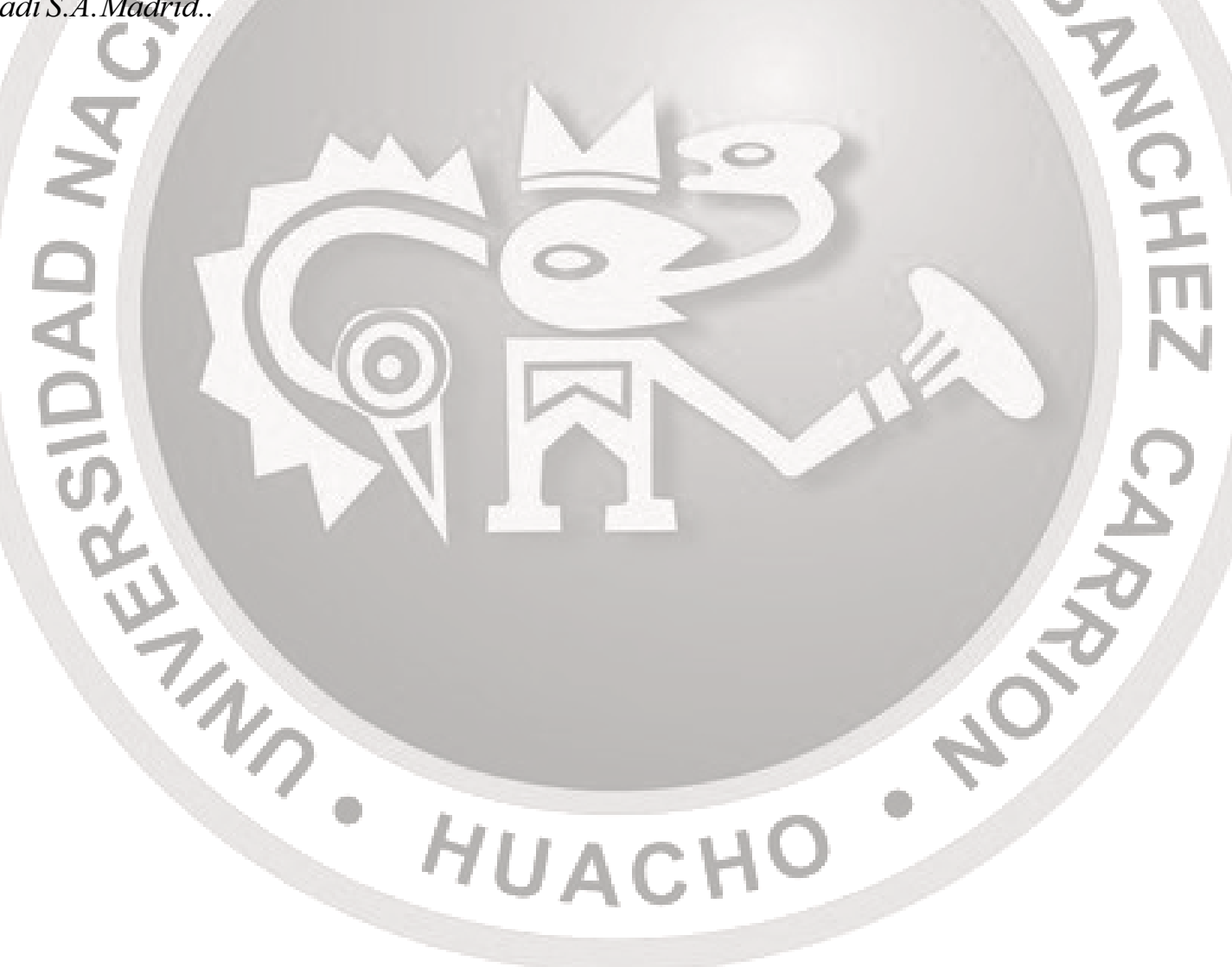

Girio Changanaqui, E. (2012. Diseño Organizacional en las Micro y Pequeñas Empresas registradas en la Cámara de comercio .

Producción de la Provincia de Huaura. (tesis de pregrado, Universidad Nacional José Faustino Sánchez Carrión-Huacho, Perú). (Acceso el 21 de noviembre del 2016).

Robbins.S Coulter. M(2014) Administracion .prentice Hall. $12^{\text {va }}$ edicion. Mexi

Rosas, N. (2016). Planeamiento estratégico y su repercusión en la gestión empresarial del sector turismo en la Región Puno 2015-2016 (tesis de maestría). Universidad Andina Néstor Cáceres Velásquez. 